Research Article

\title{
A Rapid Target Searching Scheme for a Small Satellite with Device Limits
}

\author{
Shuo Mu $\mathbb{D}^{1},{ }^{1}$ Yang Chen $\mathbb{D}^{2},{ }^{2}$ and Nan Zhang $\mathbb{D}^{1}$ \\ ${ }^{1}$ School of Aerospace Engineering, Tsinghua University, Beijing, China 100084 \\ ${ }^{2}$ General Space Engineering Department of the Second Research Institute of China Aerospace Science and Industry Corporation, \\ Beijing, China 100854
}

Correspondence should be addressed to Nan Zhang; n-zhang19@mails.tsinghua.edu.cn

Received 16 November 2020; Revised 24 January 2021; Accepted 22 February 2021; Published 15 March 2021

Academic Editor: Erkan Kayacan

Copyright (C) 2021 Shuo Mu et al. This is an open access article distributed under the Creative Commons Attribution License, which permits unrestricted use, distribution, and reproduction in any medium, provided the original work is properly cited.

In recent years, small satellites, which are more and more popular and affordable, have been already widely applied in observation and monitoring missions. However, it is a tough problem to meet the different mission requirements with such limits as space, energy, and devices. In this paper, we propose a practical target searching scheme for a small satellite which suffers from the device limits during the first 7.5 seconds after the launch separation. Due to the device limits at the beginning of the separation, the initial attitude of the satellite and the position of the target that the following observation task is based on are both unknown. In order to solve this problem, a backward integral strategy used to estimate the initial attitude and a target searching method intended to ensure the satellite acquires the target rapidly are included in the scheme. Simulation results proved that this scheme enabled the satellite, regardless of the initial conditions, to acquire the target within the limited observation time.

\section{Introduction}

Recently, small satellites have been widely applied in various missions because of their smaller size and lower cost, which makes them agiler and allows them to achieve more complex tasks [1]. On the other hand, with the limits of power and equipment, achieving the particular mission of the small satellite is a great challenge, which has been well investigated in numerous studies [2-7]. Patel et al. presented information theoretic methods for searching for a target [8]. Peng et al. developed a fast and effective Iterated Local Search algorithm for an agile earth observation satellite [9], and $\mathrm{Ci}$ et al. proposed a Gaussian distribution of target transition probability function [10], where, however, the device limits are not considered. Costic et al. described a quaternion-based control scheme for a satellite without angular velocity measurements and in the presence of an unknown inertia matrix [11]. And Wie et al. developed an attitude control system for an agile spacecraft which needed to point to the target rapidly using control moment gyros [12], which Takahashi et al. used for target pointing [13]. But there are still attitude determination sensors in their studies.

In this paper, a practical target searching scheme for a small satellite which suffers from the device limits during the first 7.5 seconds is proposed. The small satellite herein has an observation task where the satellite has to acquire the target, accurately and rapidly. But there is no power supplied during the first 7.5 seconds after the separation, during which all the attitude determination sensors will be out of work. That means the initial attitude that all the following tests are based on cannot be obtained accurately. Therefore, a numerical integral from the moment when the devices start to work back to the separation moment is addressed in this paper to estimate the initial attitude. Furthermore, a rapid target searching method is presented to ensure the satellite acquires the target within the mission time. The scheme will be of great importance to small satellites with power and device limits, ensuring them to achieve observation missions without the information from devices. 


\section{Satellite Description and Outline of Mission}

2.1. Satellite Profile. The satellite herein, working in an approximately $500 \mathrm{~km}$ sun-synchronous circular orbit, is planned to be launched recently. The satellite, with a cubic shape, has four solar arrays installed on its $-x$ face when they are folded, as Figure 1 shows.

Considering the cost and structure of the satellite, the following devices are involved for the sake of the mission:

(a) The attitude determination sensors contain two star sensors $[14,15]$, a sun sensor [16] and a magnetometer [17]

(b) The angular velocity measure device is inertial navigation system

(c) The actuators are cold gas thrusters $[18,19]$

(d) The imager [20] and laser rangefinder are used to acquire and observe the target

2.2. Mission Requirements. The task is aimed at carrying out a flight control and observation test relative to the target. Several requirements for the satellite in the test are listed as below:

(a) The speed of the satellite at the beginning of the separation ranges from $v_{1}$ to $v_{2} \mathrm{~m} / \mathrm{s}$

(b) After separation, with the $-x_{b}$ axis pointing to the target, a three-axis reorientation maneuver will be carried out so that the imager mounted on the $+x$ face, with its boresight coinciding with the inertia axis $x_{b}$, could face the target. Meanwhile, the included angle on the $z_{b}$ axis of the satellite against the vector pointing from the satellite to the geocentric should be as small as possible. The angle accuracy and the angular rate should be also considered in terms of working conditions of all the devices (the attitude determination sensors will work perfectly when the angular rate of the satellite is no more than $0.5^{\circ} / \mathrm{s}$, and the imager will make a better performance when the angle error is no more than $0.5^{\circ}$ ).

(c) After the adjustment, the satellite, holding the above pose, will be away from the target until the distance between them is approximately $H \mathrm{~m}$; then, tests such as hovering and flying-around relative to the target will follow

(d) The total time should be within 4 minutes

In order to achieve the above mentioned mission successfully, it is crucial to acquire the target within $50 \mathrm{~s}$ in the absence of the initial attitude. That is, what this paper focuses on.

\section{Satellite Dynamics Model}

\subsection{Reference Frame Definitions}

(1) The geocentric equatorial inertial J2000 coordinate system (GEI), ox $y_{i} z_{i}$ : centered in the geocentric, with

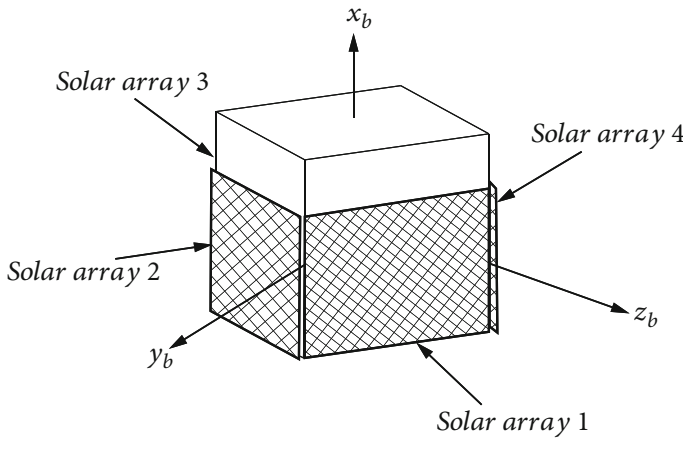

Figure 1: Diagram of the satellite.

its $x_{i}$ axis pointing toward the first point of Aries at 12 o'clock on January 1, 2000, its $z_{i}$ axis aligned with the geographic north pole and the $y_{i}$ axis completing a right-hand triad;

(2) The orbit frame, ox $y_{o} z_{0}$ : where the $z_{o}$ axis lies along the local vertical direction, pointing to the earth, the $y_{o}$ axis along a direction opposite to the orbit normal, and the $x_{o}$ axis completes a right-hand triad;

(3) The body frame, ox $y_{b} z_{b}$ : centered in the satelliteâ $€^{\mathrm{TM}} \mathrm{s}$ mass center, with its axes aligned with the inertia axes of the satellite (Figure 1).

3.2. Attitude Dynamics Model. The satellite can be regarded as a rigid body which satisfies the law of Euler equation of angular momentum:

$$
\mathbf{I} \dot{\omega}+\omega \times \mathbf{I} \omega=\mathbf{T},
$$

where I is the inertia matrix of the satellite in the body frame, $\omega$ is the angular velocity vector in the body frame with respect to the GEI frame, and $\mathbf{T}$ is the sum of the control torques and the disturbance torques in the body frame with respect to the satelliteâ $€^{\mathrm{TM}} \mathrm{s}$ mass center.

\section{Control Modes}

4.1. Initial Attitude Estimation Mode. Because of the device limits during the first 7.5 seconds after the separation, the initial attitude of the satellite can be not measured accurately by the devices. Worse still, the initial angular velocity from the environment will make great difference to the satellite during that time. Therefore, it is illogical to consider the attitude, measured when the devices are in work, as the initial attitude. An accurate estimation for this problem is proposed as follows.

As Figure 2 shows, the devices such as star sensors and inertial angular velocity sensor will be starting to work normally after the first 7.5 seconds. At that time, the full attitude and rate can be extracted accurately, which, then, will be a basis of the estimation. And before the measurement, it is necessary to damp the angular rate below $0.5^{\circ} / \mathrm{s}$ for the sake of the working conditions of the devices. Then, based on the information from the devices, the initial attitude could be obtained by the numerical integral from the moment when the devices start to work to the separation time, where 


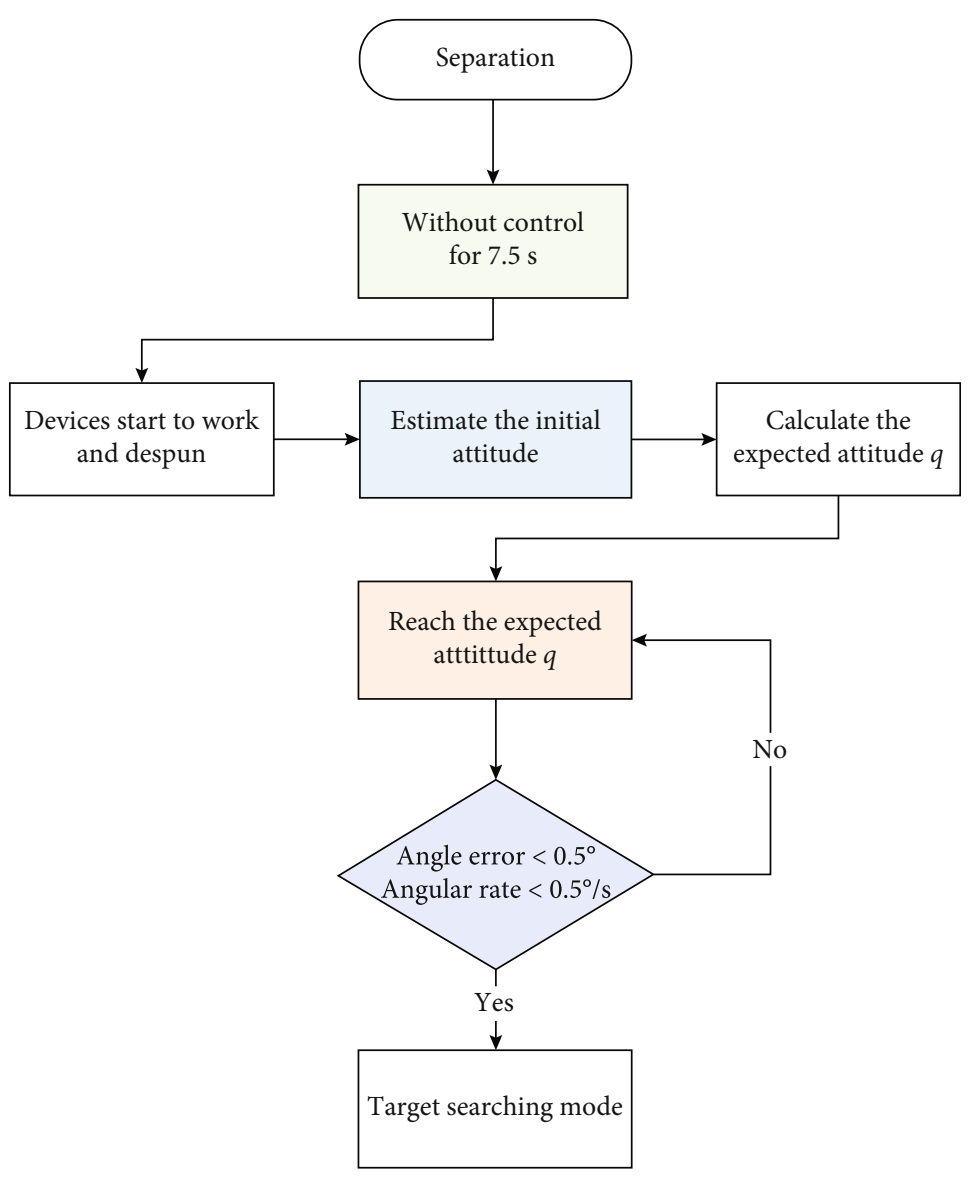

FIgURE 2: Framework of the initial attitude estimation mode.

the perturbation will be not taken into consideration since the time is too short. Furthermore, an expected attitude $q$ can be calculated where the satellite is rotated through 180 degrees around its $z_{b}$ axis on the basis of the initial attitude so that the imager could face the target directly. Finally, with the help of the propulsion system, the satellite will slew to the expected attitude with the angle accuracy better than $0.5^{\circ}$ and the angular rate better than $0.5^{\circ} \mathrm{s}$.

Note that, at the moment, the satelliteâ $€^{\mathrm{TM}} \mathrm{s} x_{b}$ axis will only point to the target roughly, so the imager may not acquire the target as the biggest initial disturbance angle is about 5 degrees, which is bigger than the field of view (FOV) of the imager (about 3 degrees). So it is necessary to develop a searching method to guarantee the imager to acquire the target.

4.2. Target Searching Mode. As mentioned earlier, after the initial attitude estimation mode, the imager still cannot point to the target precisely due to the initial disturbance angle. Luckily, a possible region where the target may exist has been confirmed in terms of the initial condition and the numerical integral, which is a cone region centered on the $x_{b}$ axis with a fixed half-cone angle of approximately 5 degrees (the region is defined in the Subsection 4.2.2 in detail). The searching method is aimed at covering all the possible region with the FOV of the imager until it captures the target. The outline of the method is as follows, which is also shown in Figure 3.
First of all, the imager can capture the target as soon as the satellite reaches the expected attitude $q$ in most cases (It is verified in the Subsection 4.2.3.). If the target is not found in the expected attitude $q$, the satellite will reorient its attitude to seven searching attitudes in turn so as to ensure a successful search. The searching attitude is defined on the basis of the expected attitude $q$, where its $x$ axis deviates from the one of the expected attitude by 4.47 degrees and the included angle between the $z$ axes of two attitudes should be as small as possible. The attitude is not unique, and the above requirements can be met by many ones. In order to cover all the possible region, seven attitudes are properly determined, whose $x$ axes are designed to be scattered uniformly on a cone which is centered on the $x$ axis of the satellite with a half-cone angle of 4.47 degrees, as Figure 4 shows. Then, with the propulsion system, the searching attitudes will be reached in turn until the target is acquired. Once the target appears in the FOV of the imager, the satellite can point its $x$ axis to the target accurately in terms of the information the imager offers with the angle accuracy better than $0.5^{\circ}$. The specific details involved in the method are as follows:

4.2.1. Searching Attitude Programming. As mentioned above, some requirements must be met for the searching attitudes in order to minimize the angle the satellite needs to rotate through. The searching attitudes could be calculated in terms of the expected attitude $q$ as follows: 


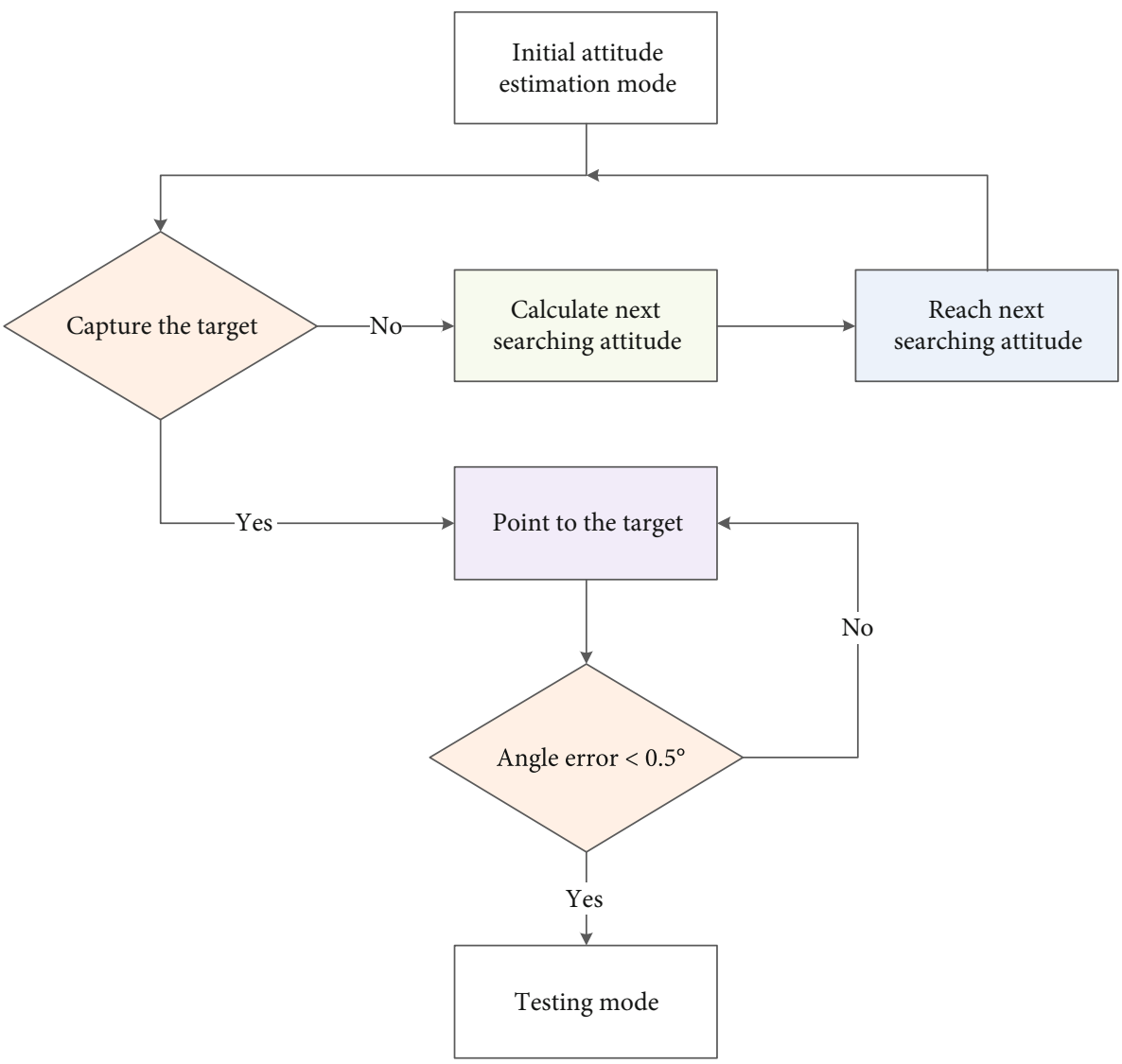

Figure 3: Framework of the target searching mode.

Firstly, a transition attitude whose $x$ axis deviates from the one of the expected attitude by 4.47 degrees is defined as follows:

$$
\mathbf{C}_{j}=\mathbf{C C}_{0} \text {, }
$$

where $\mathbf{C}_{0}$ is the matrix form of the expected attitude $q, \mathbf{C}_{j}$ is the matrix form of the transition attitude for the $j^{\text {th }}$ search $(j$ is the number of the search), and $\mathrm{C}$ is the coordinate transformation matrix between them. According to the requirements, the satellite, based on the expected attitude, has to rotate through proper angles twice to slew to the transition attitude. Hence, the coordinate transformation matrix $\mathbf{C}$ can be given from the two rotations:

$$
\mathrm{C}=\mathrm{C}_{Z} \mathrm{C}_{X}
$$

with

$$
\mathbf{C}_{x}=\left[\begin{array}{ccc}
1 & 0 & 0 \\
0 & \cos \alpha & \sin \alpha \\
0 & -\sin \alpha & \cos \alpha
\end{array}\right],
$$

$$
\mathbf{C}_{Z}=\left[\begin{array}{ccc}
\cos \beta & \sin \beta & 0 \\
-\sin \beta & \cos \beta & 0 \\
0 & 0 & 1
\end{array}\right],
$$

where $\mathbf{C}_{Z}$ and $\mathbf{C}_{X}$ are the coordinate transformation matrices corresponding to the two rotations, respectively, and $\alpha$ and $\beta$ are the angles the satellite needs to rotate through, whose values are

$$
\begin{aligned}
& \alpha=\frac{(j-1) 2 \pi}{7}, \\
& \beta=\frac{4.47 \pi}{180} .
\end{aligned}
$$

Considering the included angle between the $z$ axes of searching attitudes and the expected attitude should be as small as possible, finally, the three axes of the searching attitude for the $j^{\text {th }}$ search in $o x_{i} y_{i} z_{i}$ could be calculated from

$$
\begin{aligned}
& i_{x}=i_{X}, \\
& i_{y}=i_{Z} \times i_{x}, \\
& i_{z}=i_{x} \times i_{y},
\end{aligned}
$$




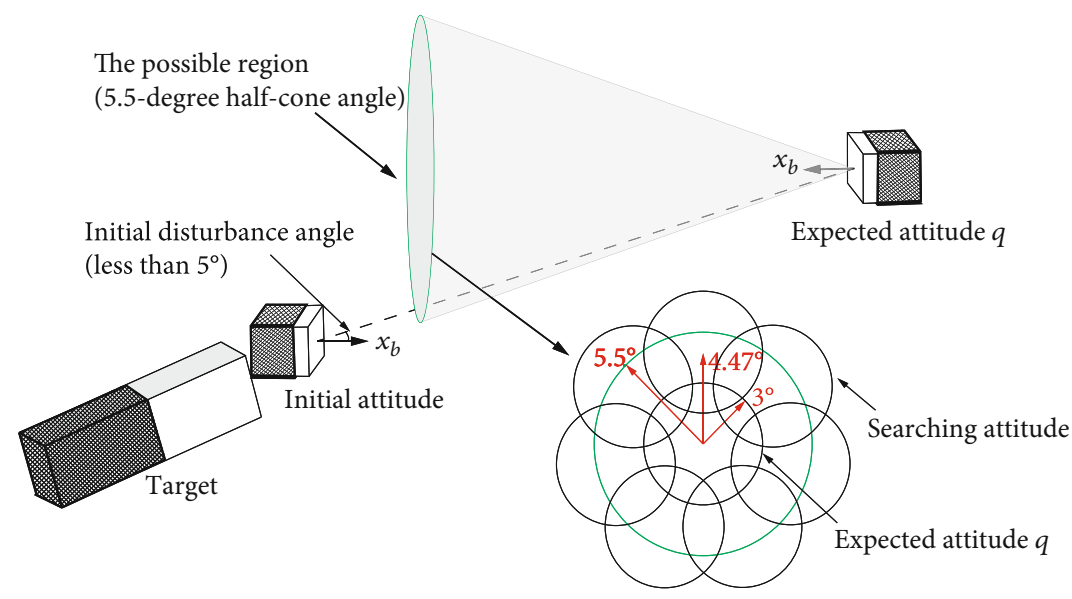

FIGURE 4: Diagram of target searching scheme.

where $i_{X}$ and $i_{Z}$ are unit vectors of the $x$ and $z$ axes of the transition attitude, respectively.

4.2.2. Searching Scheme and Possible Region. As was mentioned before, the initial attitude can be obtained by the integral in terms of the attitude and angular rate measured by the devices, based on which the expected attitude $q$ will be calculated. As Figure 4 shows, in the expected attitude $q$, the satellite is capable of pointing its $x_{b}$ axis to the target roughly. But there is still a deviation because of the initial disturbance angle (less than 5 degrees). It is assumed that the satellite will be away from the target straightly along the direction of separation speed since the device limit time is too short to make great difference to the path. Therefore, the deviation should be less than 5 degrees, theoretically. In consideration of the motion perpendicular to the path during maneuvering (less than $0.1 \mathrm{~m}$ ), finally, the deviation will be no more than 5.5 degrees. So a cone region centered on the $x_{b}$ axis with a half-cone angle of 5.5 degrees is regarded as the possible region where the target may appear. The method is aimed at covering all the possible region with the FOV of the imager.

The FOV of the imager is also a cone region with a halfcone angle of 3 degrees. That is, when the satellite reaches the expected attitude, a cone region centered on its $x_{b}$ axis with a half-cone angle of 3 degrees would be visible. But it is not easy for the satellite to slew to the particular attitude accurately during such a short time, which means the FOV of the imager should be smaller than the theoretical one. To be on the safe side, a 0.5 -degree attitude error should be taken into account (about 0.2 degrees in fact) and the visible region, finally, is a cone with a half-cone angle of 2.5 degrees. It is obvious that what we need to do is to cover the possible region with the cone region with a half-cone angle of 2.5 degrees.

As is shown, the most effective method is to unite the expected attitude $q$ with the seven searching attitudes (they are calculated in the Subsection 4.2.1), which could be well verified. The satellite will reach the expected attitude $q$ first and search for the target. Then, the searching attitudes will be reached in sequence to look for the target. By doing so, the target will be acquired in one of the above poses for sure.
4.2.3. Probability of the First Search. As a matter of fact, it is very possible to bring down the target at the first time. The probability that the target is acquired in the expected attitude $q$ is given as follows:

It is mentioned earlier that the initial disturbance angle and error during maneuvering are 5.5 degrees, totally, which can be described in quaternion form as follows:

$$
\mathbf{q}=e_{x} \sin \frac{\theta}{2} i+e_{y} \sin \frac{\theta}{2} j+e_{z} \sin \frac{\theta}{2} k+\cos \frac{\theta}{2},
$$

where $\theta \in[05.5]^{\circ}, e_{x}, e_{y}$, and $e_{z}$ are unit vectors distributing evenly on the unit sphere, which can be represented by the angles $\alpha$ and $\beta$ as follows:

$$
\begin{gathered}
e_{x}=\sin \beta, \\
e_{y}=\cos \alpha \cos \beta, \\
e_{z}=\sin \alpha \cos \beta,
\end{gathered}
$$

where $\alpha \in\left[\begin{array}{ll}0 & 2 \pi]\end{array}\right]$ and $\beta \in[-\pi / 2 \pi / 2]$ with $\cos \beta / 2$ as the probability density function. According to the Equation (7), the included angle $\gamma$ of the $x_{b}$ axis before and after the disturbance should meet the requirement:

$$
\cos \gamma=e_{x}^{2} \sin ^{2} \frac{\theta}{2}-e_{y}^{2} \sin ^{2} \frac{\theta}{2}-e_{z}^{2} \sin ^{2} \frac{\theta}{2}+\cos ^{2} \frac{\theta}{2} \text {. }
$$

Substituting Equations (8), (9), and (10) into Equation (11), Equation (11) could be described as follows:

$$
\cos \beta=\frac{\sin \gamma / 2}{\sin \theta / 2}
$$

Restricted by the boundary condition

$$
1=\int_{0}^{A} \int_{-\pi / 2}^{\pi / 2} \frac{\cos \beta}{2 A} d \beta d \theta
$$


with $A$ being a constant number, whose value is $5.5 \times \pi /$ 180, substituting Equation (12) into Equation (13), Equation (13) could be described as follows:

$$
1=\int_{0}^{A} \int_{\gamma}^{A} \frac{\sin \gamma}{4 A \sin \theta / 2 \sqrt{\sin ^{2} \theta / 2-\sin ^{2} \gamma / 2}} d \theta d \gamma
$$

Ultimately, the probability density function $f(\gamma)$ could be given from

$$
f(\gamma)=\int_{\gamma}^{A} \frac{\sin \gamma}{4 A \sin \theta / 2 \sqrt{\sin ^{2} \theta / 2-\sin ^{2} \gamma / 2}} d \theta .
$$

According to the probability density function $f(\gamma)$, the probability function $F(\gamma)$ could be donated. And it can be calculated that the value of $F(\gamma)$ is about 0.70 when $\gamma$ is 3 degrees, which means it is $70 \%$ likely to capture the target once the satellite reaches the expected attitude $q$. Therefore, the scheme is able to ensure the satellite acquires the target as soon as possible.

4.3. Testing Mode. After captures the target, the satellite will get away from the target until reaches a particular position. Then, according to the position of the target, the tests, such as hovering and flying-around, will be carried out. Finally, as all the tests are completed, the satellite will unfold the solar arrays, adjust the attitude, point its $x_{b}$ axis to the sun, and hold the pose all along, waiting for the next command from the earth.

\section{Simulation}

A numerical simulation for the scheme has come out, and the initial states are listed as follows: the satellite will come into work in a $550 \mathrm{~km}$ sun-synchronous circular orbit whose inclination is $99.8^{\circ}$, at the midnight on January 1, 2019. The initial attitude is the same as the orbit frame with a less than 5 -degree initial disturbance angle. The separation speed ranges from $v_{1}$ to $v_{2}$ randomly along the tangent to the orbit. And the separation angular rate around body frame axes is no more than $10^{\circ} / \mathrm{s}$ with a random direction. The inertia matrix of the satellite is

$$
\mathbf{I}=\left[\begin{array}{ccc}
6.38 & -0.07 & 0.07 \\
-0.07 & 8.86 & 0.33 \\
0.07 & 0.33 & 8.81
\end{array}\right]\left(\mathrm{kg} / \mathrm{m}^{2}\right)
$$

5.1. Normal Initial Condition. The separation speed is $1.2 \mathrm{~m} / \mathrm{s}$, the initial disturbance angle is $3.8^{\circ}$.

The angular rate during all modes is shown in Figure 5 where the satellite completes all the tests successfully. As shown in Figure 6, the total time of the initial attitude estimation mode and target searching mode is $21.9 \mathrm{~s}$ where the tar-

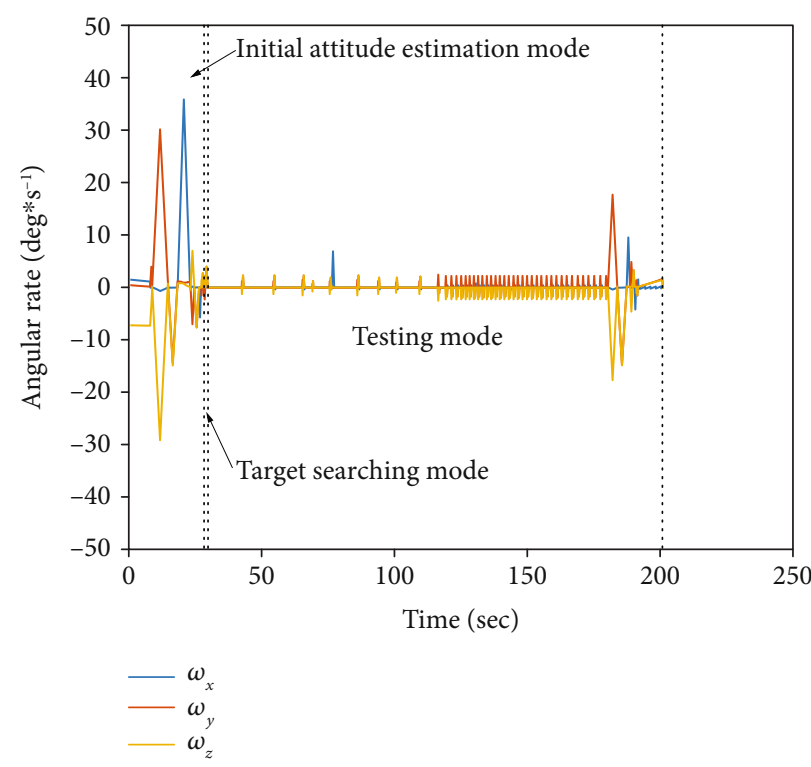

FIGURE 5: Time history of angular rate during all modes.

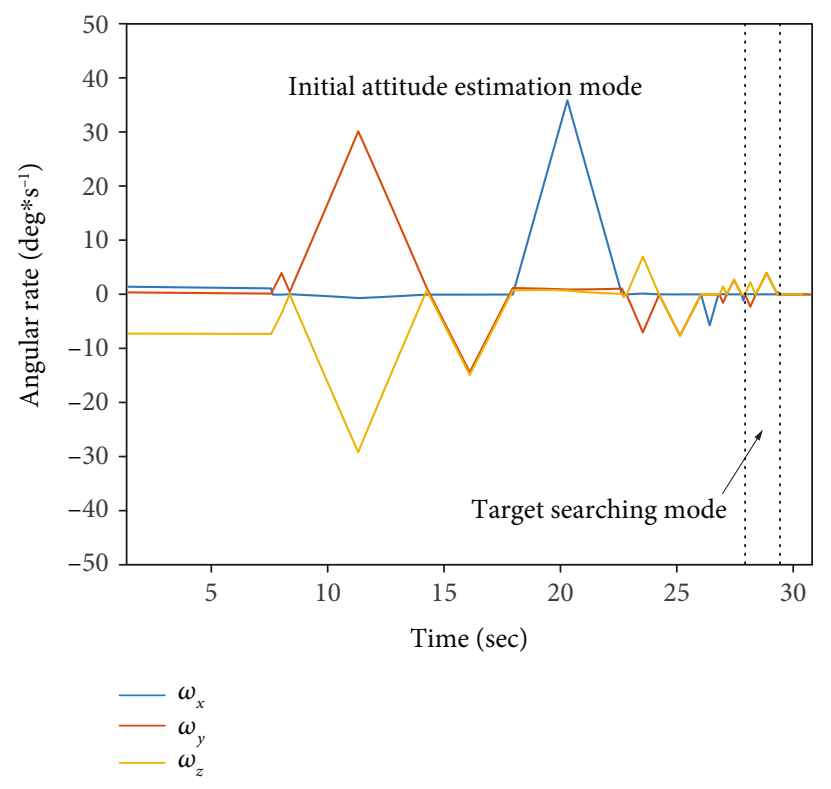

FIgURE 6: Time history of angular rate during initial attitude estimation mode and target searching mode.

get searching mode just lasts for $1.5 \mathrm{~s}$, which is much shorter than the schedule time. In the initial attitude estimation mode, the satellite has to slew into the expected attitude $q$ rapidly and point its $x_{b}$ axis to the target. Therefore, there are great changes of angular rate in this mode which results from the attitude maneuver. Then, the satellite stabilizes gradually, making a fully preparation for the target searching. Figure 7 shows the time history of the satellite attitude during the two modes where the satellite gives a good performance in maintaining the attitude in the target searching mode. That means there is no maneuver for the satellite to reach the searching attitudes during this mode, and it acquires the target at the first time, confirming the feasibility and efficiency of the scheme. 


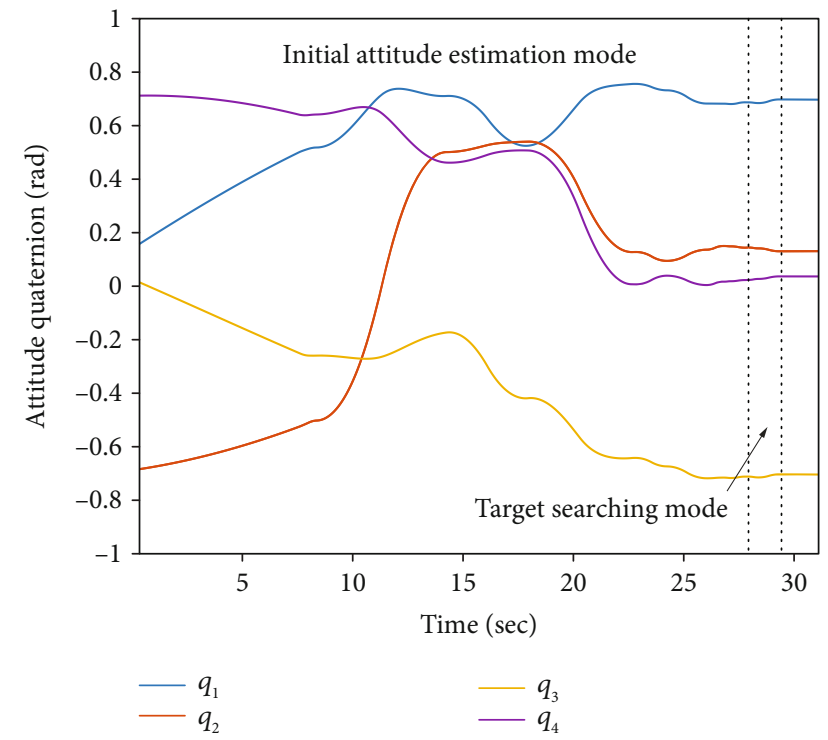

FIGURE 7: Time history of attitude quaternion during initial attitude estimation mode and target searching mode.

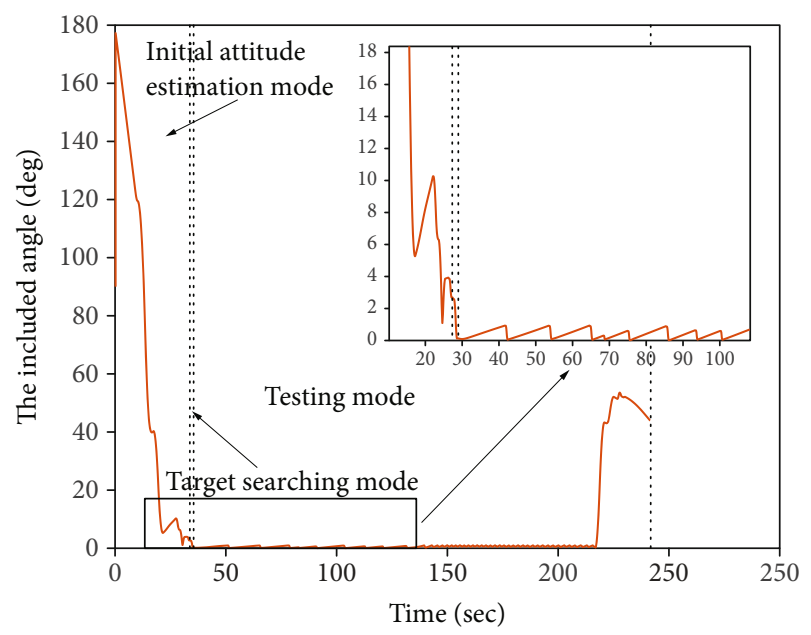

FIGURE 8: Time history of the included angle between the satelliteâ $€^{\mathrm{TM}} \mathrm{s} x$ axis and the array pointing from the satellite to the target during all modes.

The time history of the included angle between the satelliteâ $\epsilon^{\mathrm{TM}} \mathrm{s} x_{b}$ axis and the array pointing from the satellite to the target is shown in Figure 8. Similarly, because of the attitude adjustment, there is also a shape decrease in the angle, from $180^{\circ}$ to about $0^{\circ}$, in agreement with the change of the angular rate. That also illustrates that the satellite points its $x_{b}$ axis to the target successfully. And at the end of the searching, the angle has decreased almost to vanishing point, satisfying the point requirements well.

5.2. Worst Initial Condition. In order to confirm the feasibility of the scheme further, a simulation under the worst initial condition has been run where the separation speed is $2 \mathrm{~m} / \mathrm{s}$ and the initial disturbance angle is $5^{\circ}$. The results are as follows:

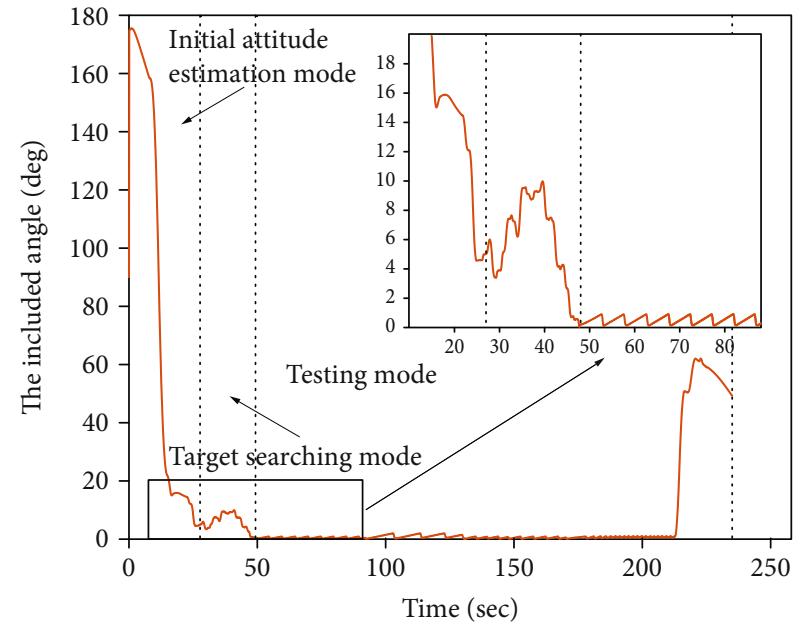

FIgURE 9: Time history of the included angle between the satelliteâ $€^{\mathrm{TM}} \mathrm{s} x$ axis and the array pointing from the satellite to the target during all modes.

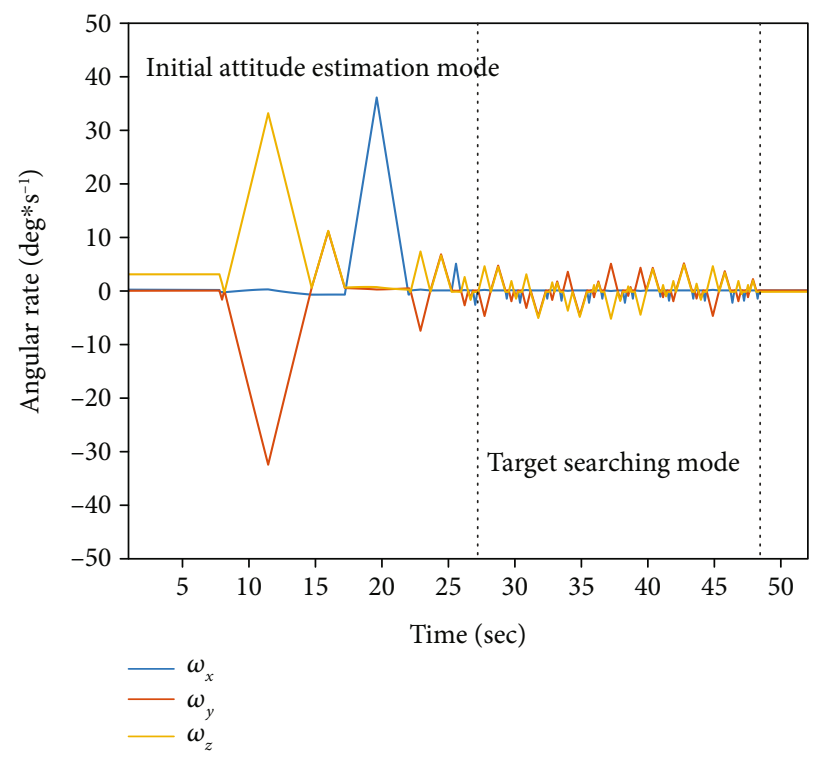

FIgURE 10: Time history of angular rate during initial attitude estimation mode and target searching mode.

Compared with the first simulation, the total time of the two modes under the worst initial condition, obviously, is much longer: the estimation mode lasts for $19.5 \mathrm{~s}$, and the searching mode lasts for $21.2 \mathrm{~s}$, but it is still within the schedule time. There is no significant change in the initial attitude estimation mode where the satellite is still capable of pointing its $x_{b}$ axis to the target quickly but a shape increase has been seen in the target searching mode. As shown in Figure 9, there are seven great fluctuations in the searching mode, in consistency with the seven attitude adjustments to reach the searching attitudes, which results in the great increase of the searching time. The attitude maneuvers also result in the frequent fluctuations in Figures 10 and 11. Further, it can be seen that at the end of the searching, the included angle between the satelliteâ $\mathrm{E}^{\mathrm{TM}} \mathrm{s} x$ axis and the array pointing 


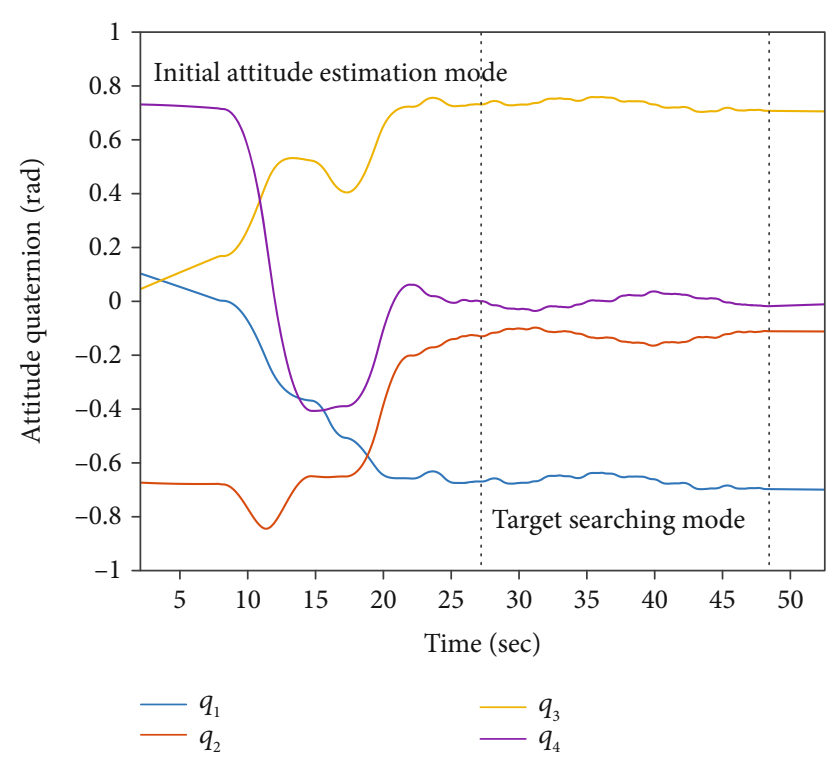

FIGURE 11: Time history of attitude quaternion during initial attitude estimation mode and target searching mode.

TABLE 1: Total mission time of both initial attitude estimation mode and target searching mode in different initial condition settings.

\begin{tabular}{|c|c|c|c|c|}
\hline Initial disturbance angles & NAM & $2 \% \mathrm{~s}$ & $5 \% \mathrm{~s}$ & $10^{\circ} / \mathrm{s}$ \\
\hline $2^{\circ},\left[\begin{array}{lll}0 & 0 & -1\end{array}\right]$ & 0 & $24.3 \mathrm{~s}$ & $23.7 \mathrm{~s}$ & $23.4 \mathrm{~s}$ \\
\hline $5^{\circ},\left[\begin{array}{lll}0 & 0 & -1\end{array}\right]$ & 1 & $26.4 \mathrm{~s}$ & $26.0 \mathrm{~s}$ & $25.6 \mathrm{~s}$ \\
\hline $5^{\circ},\left[\begin{array}{lll}0 & -0.78 & -0.62\end{array}\right]$ & 2 & $28.8 \mathrm{~s}$ & $28.5 \mathrm{~s}$ & $28 \mathrm{~s}$ \\
\hline $5^{\circ},\left[\begin{array}{lll}0 & -0.97 & 0.22\end{array}\right]$ & 3 & $31.9 \mathrm{~s}$ & $31.3 \mathrm{~s}$ & $31.3 \mathrm{~s}$ \\
\hline $5^{\circ},\left[\begin{array}{lll}0 & -0.43 & 0.90\end{array}\right]$ & 4 & $34.0 \mathrm{~s}$ & $33.7 \mathrm{~s}$ & $33.5 \mathrm{~s}$ \\
\hline $5^{\circ},\left[\begin{array}{lll}0 & 0.43 & 0.90\end{array}\right]$ & 5 & $36.5 \mathrm{~s}$ & $36.6 \mathrm{~s}$ & $36.2 \mathrm{~s}$ \\
\hline $5^{\circ},\left[\begin{array}{lll}0 & 0.97 & 0.22\end{array}\right]$ & 6 & $39.3 \mathrm{~s}$ & $38.4 \mathrm{~s}$ & $38.8 \mathrm{~s}$ \\
\hline $5^{\circ},\left[\begin{array}{lll}0 & 0.78 & -0.62\end{array}\right]$ & 7 & $41.9 \mathrm{~s}$ & $41.3 \mathrm{~s}$ & $41.4 \mathrm{~s}$ \\
\hline
\end{tabular}

from the satellite to the target also approaches zero, demonstrating the validity of the scheme under the worst condition.

In a word, although the initial condition is much worse in this simulation, the satellite is still able to acquire the target effectively.

5.3. Comparison in Different Cases. Table 1 compares the total time of the two modes in 24 cases where the separation angular rates are $2 \%, 5 \%$, or $10^{\circ} \%$, respectively, and the numbers of the attitude maneuver(NAM) range from 0 to 7 with different initial disturbance angles. The direction vector of the separation angular rates is always set as $[0.300 .75-0.60]$ in the following simulations, which is selected randomly.

As the Table 1 shown, regardless of the initial condition, the satellite could complete the target searching mission within $50 \mathrm{~s}$, all of which meet the requirement for the searching time. Furthermore, the total time also shows an upward trend with the increase of NAM, which is because more attitude maneuvers for searching target need more time. Even in the case of 7 attitude maneuvers, the total mission time is only about $41 \mathrm{~s}$, fast less than $50 \mathrm{~s}$. But it is not the case with the separation angular rates: there is no significant change for the searching time when the rates change.

\section{Conclusion}

A rapid target searching scheme for a small satellite, suffering from device limitation during the first $7.5 \mathrm{~s}$ after the separation, has been proposed in this paper. In order to deal with the device limits, a backward integral from the moment when the devices start to work to the separation moment, on the basis of the information measured by the devices, is utilized to estimate the initial attitude. Then, based on the initial attitude, a searching method is addressed which can ensure the satellite acquires the target regardless of the initial condition. A probability density function has been presented so as to confirm it is very likely that the satellite captures the target once it reaches the expected attitude and demonstrates the efficiency of the scheme, which is also verified by the simulation results. What is more, the searching scheme has been applied to a small satellite planned to be launched recently, which will demonstrate the reliability of the scheme further. The scheme will make great difference to small satellites suffering from power and device limits, ensuring them to achieve observation or monitoring missions without attitude and angular velocity for a quiet time. But the searching region of the current scheme is only a cone region with a half-cone angle of approximately 5 degrees. In the future, a rapid searching scheme with a larger searching region can be developed, which will be of value to small satellites with more complex observation missions.

\section{Data Availability}

No data used to support the study.

\section{Conflicts of Interest}

The authors declare that there is no conflict of interest regarding the publication of this paper.

\section{References}

[1] L. Zhen, "Status and trends of the small satellite and micronano satellites," Journal of Ordnance Equipment Engineering, vol. 39, no. 6, pp. 1-7, 2018.

[2] K. Tawara and S. Matunaga, "On attitude control of microsatellite using shape variable elements," in the 25th workshop on JAXA: Astrodynamics and flight mechanics, pp. 1-5, Sagamihara, Japan, 2015.

[3] M. Fritz, J. Shoer, L. Singh et al., "Attitude determination and control system design for the CYGNSS microsatellite," in 2015 IEEE Aerospace Conference, pp. 1-12, Big Sky, MT, USA, 2015.

[4] A. S. Mohammed, "An attitude determination and control system of the Alsat-1 first Algerian microsatellite," in International Conference on Recent Advances in Space Technologies, 2003. RAST '03, pp. 171-176, Istanbul, Turkey, 2003.

[5] K. Han, H. Wang, and Z.-h. Jin, "Magnetometer-only linear attitude estimation for bias momentum pico-satellite," Journal of Zhejiang University-SCIENCE A, vol. 11, no. 6, pp. 455-464, 2010. 
[6] T. Hao and S. Matunaga, "A practical attitude control system using control moment gyros for nano-satellite tsubame," in 53rd AIAA Aerospace Sciences Meeting, p. 0940, Kissimmee, Florida, USA, 2015.

[7] G. Avanzini and F. Giulietti, "Magnetic detumbling of a rigid spacecraft," Journal of Guidance, Control, and Dynamics, vol. 35, no. 4, pp. 1326-1334, 2012.

[8] M. Patel, A. J. Sinclair, and K. Ho, "Information-theoretic target search for space situational awareness," in 2018 Space Flight Mechanics Meetingp. 0725, 2018.

[9] G. Peng, P. Vansteenwegen, X. Liu, L. Xing, and X. Kong, “An iterated local search algorithm for agile earth observation satellite scheduling problem," in 2018 SpaceOps Conference, p. 2311, Marseille, France, 2018.

[10] Y.-z. Ci, R.-j. He, Y.-f. Xu, and Y.-j. Tan, "Method of target motion prediction for moving target search by satellite," Control and Decision, vol. 24, no. 7, pp. 1007-1012, 2009.

[11] B. T. Costic, D. M. Dawson, M. S. de Queiroz, and V. Kapila, "Quaternion-based adaptive attitude tracking controller without velocity measurements," Journal of Guidance, Control, and Dynamics, vol. 24, no. 6, pp. 1214-1222, 2001.

[12] B. Wie, D. Bailey, and C. Heiberg, "Rapid multitarget acquisition and pointing control of agile spacecraft," Journal of Guidance, Control, and Dynamics, vol. 25, no. 1, pp. 96-104, 2002.

[13] M. Takahashi, Y. Nanamori, and K. Yoshida, "Rapid multitarget pointing and high accuracy attitude control steering law of variable speed control moment gyroscopes," in AIAA Guidance, Navigation and Control Conference and Exhibit, p. 7015, Honolulu, Hawaii, USA, 2008.

[14] W. Hong-tao, L. Chang-zhou, W. Yu, and Z. Shu-fang, "Star sensor model parametric analysis and calibration method study," Journal of the University of Electronic ence and Technology of China, vol. 39, no. 6, pp. 880-885, 2010.

[15] W. Yong-sheng, W. Hong-li, L. Jie-liang, and C. Xiang-xiang, "Error model and parametric analysis of star sensor," Electronics Optics \& Control, vol. 21, no. 2, pp. 85-95, 2014.

[16] C. W. de Boom, J. A. P. Leijtens, L. M. H. V. Duivenbode, and N. van der Heiden, "Micro digital sun sensor: System in a package," in 2004 International Conference on MEMS, NANO and Smart Systems (ICMENS'04), pp. 322-328, Banff, AB, Canada, 2004.

[17] X. U. Jian and L. I. Run, "Analytical calculation of distribution and gradient of magnetic field in separation space of doubleroll high-intensity magnetic separator," Mining and Metallurgy, vol. 155, no. 6, pp. 999-1001, 2001.

[18] R. Cocomazzi, A. Avanzi, D. Modenini, and P. Tortora, "Systems design and performance of cold gas microthruster for microsatellite attitude control," in 42nd AIAA/ASME/SAE/ASEE Joint Propulsion Conference \& Exhibit, p. 4629, Sacramento, California, USA, 2006.

[19] N. A. Gatsonis, L. Ye, J. J. Blandino, M. Demetriou, and N. Paschalidis, "Cubesat design and attitude control with micro pulsed plasma thrusters," in AIAA SPACE 2014 Conference and Exposition, p. 4211, San Diego, California, USA, 2014.

[20] L. J. Otten III, A. D. Meigs, B. Al Jones et al., "Engineering model for the MightySat II. 1 hyperspectral imager," in Sensors, Systems, and Next-Generation Satellites, pp. 412-420, International Society for Optics and Photonics, 1997. 